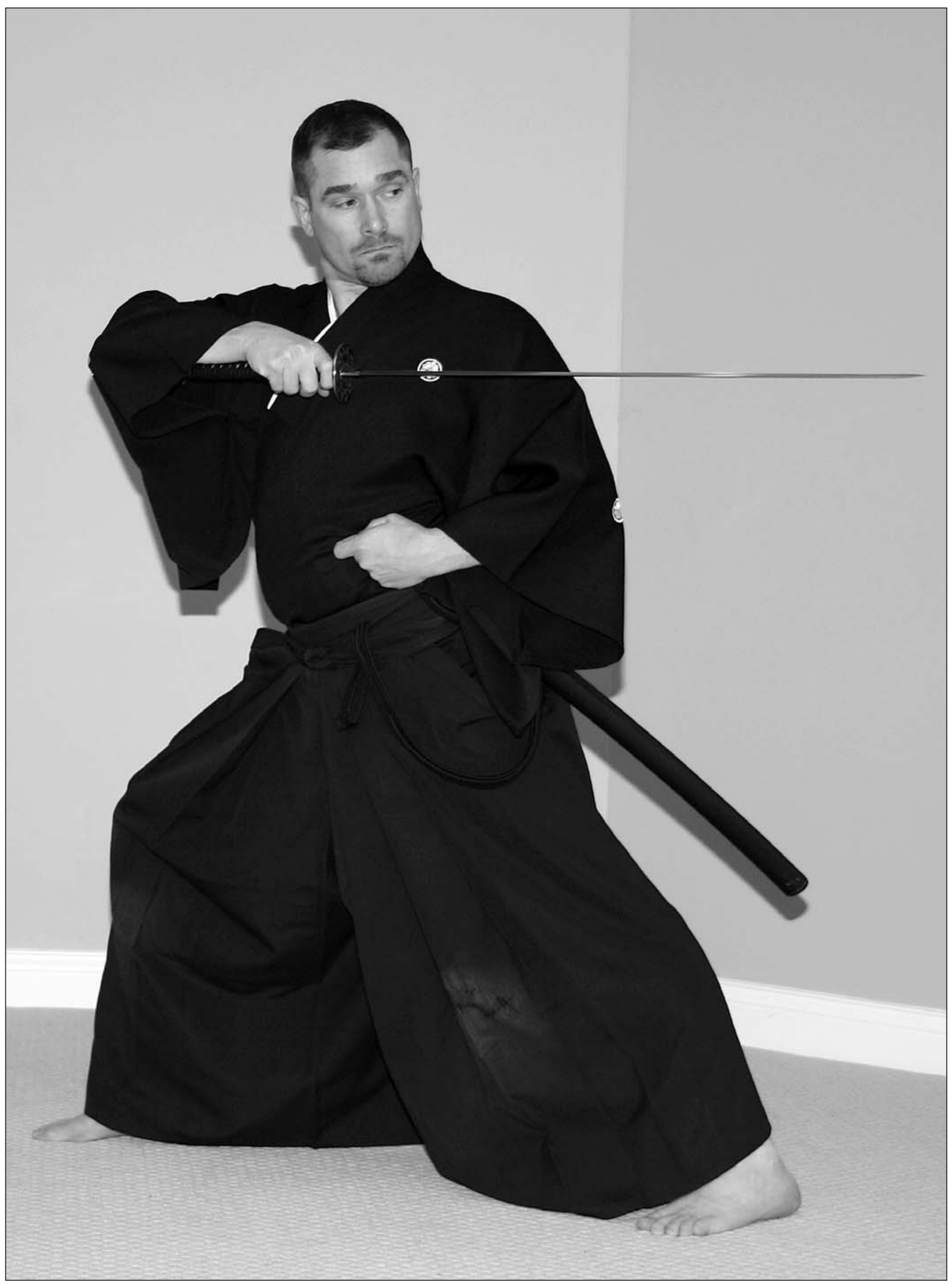




\section{El agarre adecuado de la espada en el Iaido Muso Jikiden Eishin-ryu*}

Nicklaus Suino

\section{Introducción}

Pocas artes presentan tantos retos en la ejecución de sus detalles como lo hace el iaido. Cada movimiento está cuidadosamente coreografiado en orden a lograr un uso de la anatomía humana altamente efectivo, un eficiente fluir de la energía y una belleza técnica y económica. El iaido realizado por un verdadero experto parece sencillo, pero cada golpe de espada está compuesto, realmente, por muchos detalles cruciales. Sólo hay unos pocos practicantes del arte que tengan el entrenamiento, el carácter y el tiempo de práctica para dominar todos los detalles hasta el grado necesario para lograr una auténtica maestría. La mayor parte de los practicantes de iaido fuera de Japón, y desafortunadamente muchos en Japón, practican gran parte de su arte sin una máxima eficiencia.

Entre los aspectos del Iaido Eishin-ryu que habitualmente se ejecutan incorrectamente figura el agarre adecuado de la empuñadura del iaito (espada de práctica). A esto se le denomina "tenouchi", que literalmente significa "dentro de las manos". El agarre es fundamental porque es el punto central final en la transmisión de fuerza desde el cuerpo, a través de los brazos, y el filo de la espada. Un agarre correcto también proporciona "sensación", que incrementa la conciencia de la posición de la espada y ayuda a guiar el filo por el camino correcto en los cortes. Si el agarre es incorrecto durante el corte principal en cada forma (kirioroshi), el corte no alcanzará su fuerza potencial, la espada temblará excesivamente al final del filo y el estudiante tenderá subconscientemente a desvirtuar el corte permitiendo que sus manos y brazos actúen suavemente justo cuando finaliza dicho corte. En los cortes de prueba (tameshigiri), un agarre pobre dará como resultado fallar al tratar de cortar limpiamente el objetivo.

\section{El corte descendente}

He escrito en algún otro lugar sobre los principales elementos de un buen y directo corte descendente (véase Suino, 1994), por lo tanto aquí será suficiente una rápida revisión para reintroducir de nuevo el tema al lector. En lo más alto del corte, la espada se sostiene sobre la cabeza con la hoja descendiendo hacia atrás en un ángulo de 45 grados. Los codos están abiertos y las bases de las manos están lejos de la empuñadura (tsuka). Según comienza el corte los brazos se extienden y los codos comienzan a ponerse rectos, provocando que las muñecas roten hacia adentro. Aproximadamente sobre un tercio del arco que describe el golpe, los brazos deberían estar rectos y las palmas deberían estar en la parte superior de la empuñadura. Durante los últimos dos tercios del corte los brazos se mantienen rectos y los hombros hacia abajo. La espada debe realizar un gran arco, llegando a descansar en la

\section{* Nota:}

Muso se refiere a Hayashizaki Junsuke (aprox. 1542-1621), el fundador de este sistema que "no tiene igual bajo el cielo". Jikiden significa "transmisión directa”. Eishin se refiere a Hasegawa Chikaranosuke Eishin, el nombre del $7^{\circ}$ Maestro del estilo. Se dice que el estilo tiene más de 450 años de historia y es una de las artes marciales más antiguas que existen actualmente en Japón.

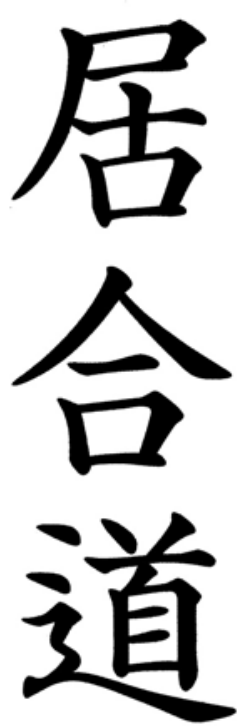

(página anterior)

Suino ejecuta la estocada inicial en Tsuredachi (Acompañante) desde la posición avanzada de pie.

Todas las fotografías son cortesía de Niklaus Suino. 


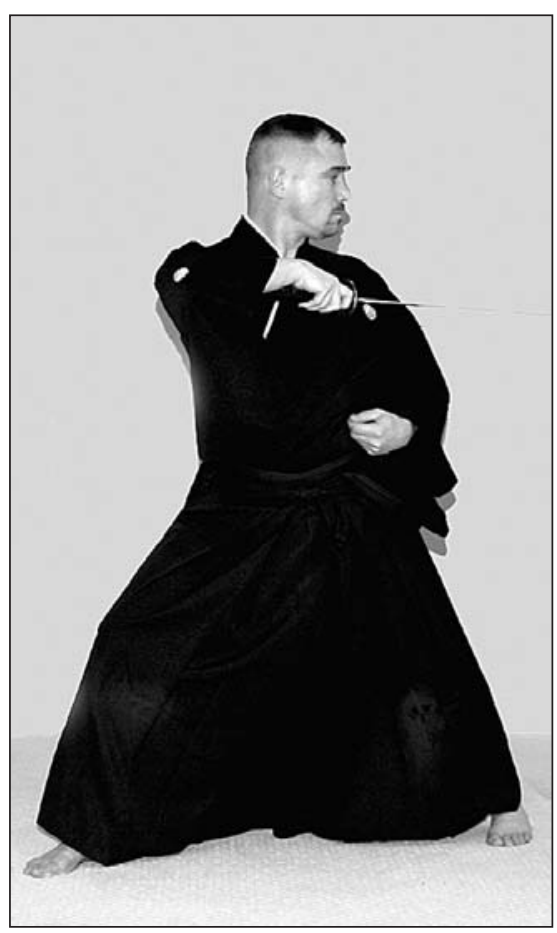

(fotografía superior izquierda) En Tatekito

(corte multidireccional), del Conjunto de los Métodos de la Espada, la mirada del espadachín debe preceder a la estocada hacia la esquina posterior izquierda.

(fotografía superior centro)

Una interpretación del movimiento de apertura de Tatehiza's

Oroshi (Postura Medio-Sentada Viento de Montaña), es un bloqueo de muñeca contra un oponente que agarra la empuñadura de nuestra espada.

(fotografía superior derecha) En Yukizure (Acompañamiento), el corte de apertura se dirige hacia las muñecas

del oponente para pararle en el intento de sacar su espada.
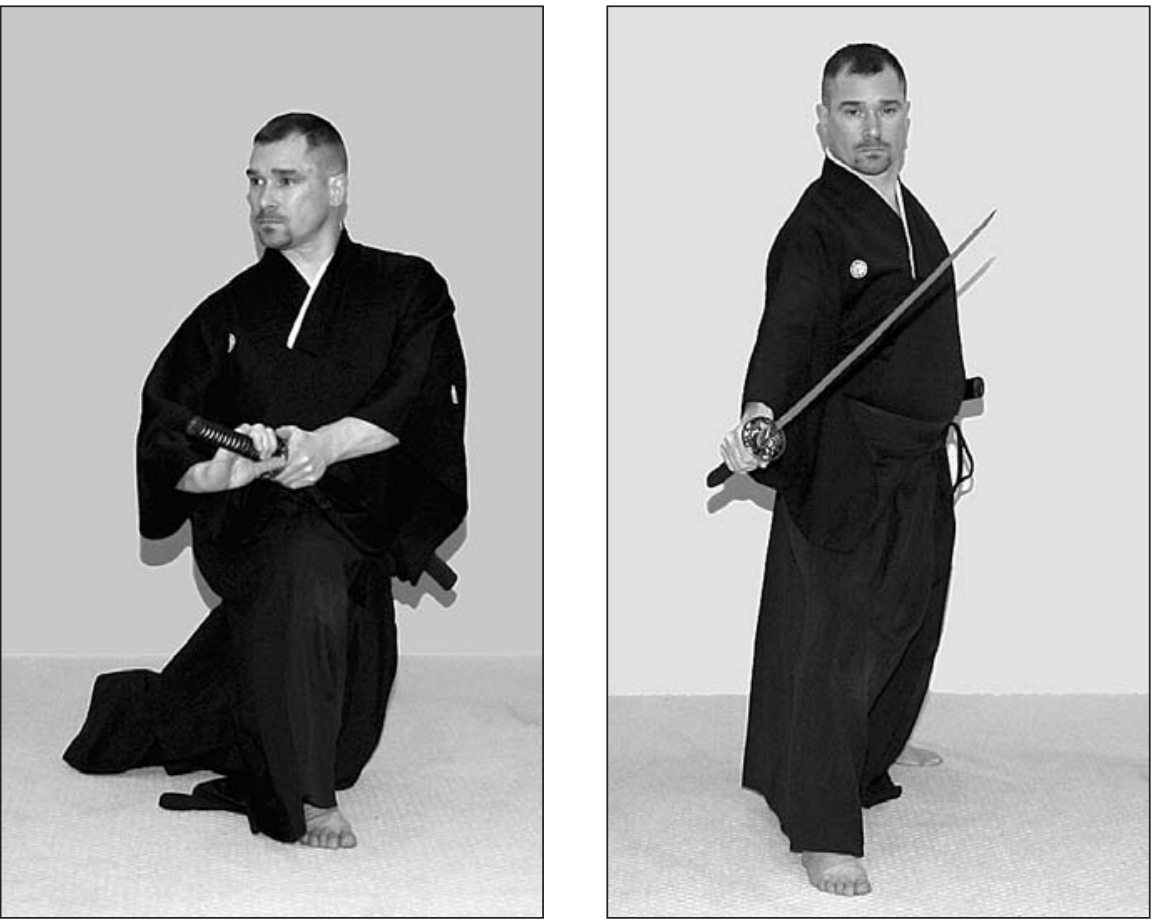

parte trasera de la empuñadura (tsukagashira) a un puño de distancia del abdomen. Todos los movimientos deben finalizar en seco al final del corte. La hoja debe finalizar casi al nivel, con la punta (kissaki) a la misma altura o ligeramente más baja que la empuñadura. Se acepta alguna variación en el ángulo final de la espada, pero esto se debe a la diferente construcción de cada espada -tal como el ángulo de la espiga (nakago) o la forma de la empuñadura- más que a variaciones en los parámetros correctos para realizar el agarre.

\section{Agarrando la espada}

Existen muchos aspectos que constituyen el buen agarre de la espada, algunos de los cuales no son técnicamente parte del te-no-uchi, tales como la distancia entre las manos, la rotación de las manos durante el corte o la posición de las muñecas. Otros aspectos del agarre son parte claramente del te-no-uchi, tales como la posición de la empuñadura en las palmas de las manos, la posición extendida de las manos en la empuñadura, la sensación de los dedos durante el corte y la fuerza del corte. Su propósito es proporcionar los medios más eficientes para dirigir la fuerza física desde los brazos, a través de las manos y hasta el filo de la espada. Todos son importantes y deben ser cuidadosamente estudiados y practicados a fondo por los estudiantes de iaido.

\section{Distancia entre las manos}

Las manos deben colocarse tan lejos una de otra como sea posible, mientras se mantienen todos los dedos de la mano izquierda sobre la empuñadura. Aunque usted pueda leer sobre otros estilos de esgrima que animan a los estudiantes a colocar la mano izquierda de modo que uno o más dedos se sitúan fuera del final de la empuñadura, esto no es apropiado para el Iaido Eishin-ryu. De hecho, este autor ha 
entrenado y competido contra practicantes de muchos linajes de esgrimistas japoneses, incluyendo Muso Shinden-ryu, Hoki-ryu, Suio-ryu, Katori Shinto-ryu, Mugai-ryu, y Jigen-ryu, y todavía no ha encontrado a un profesor que señale a sus estudiantes que agarren la empuñadura con menos de la totalidad de los diez dedos. La razón de esto es obvia: más dedos en la empuñadura proporcionarán más fuerza y control de la hoja. Por tanto, mientras que pueden existir estilos de esgrima que dejan uno o varios dedos de la mano izquierda fuera de la empuñadura, usted no debería realizar este tipo de agarre durante su práctica a menos que no sea aconsejado específicamente por su profesor. La mano derecha debería estar tan cerca como pueda de la guarda (tsuba) (teniendo en cuenta la posición extendida de las manos señalada más arriba), de modo que el primer nudillo del dedo índice toque la guarda. Si el agarre es correcto, el nudillo se inflamará frecuentemente (y con el tiempo le saldrá callo) debido a la textura de la guarda.

El espacio entre las manos proporciona un grado de efecto de palanca y control que no es posible si las manos están juntas. En esto se distingue del agarre de un palo de golf o de un bate de béisbol, donde las manos están juntas, o del agarre de un hacha, que es apropiado para golpear. Tanto en el golf como en el béisbol se pretende que el palo o el bate se balanceen libremente en el momento del impacto para transmitir energía a la pelota. Con un hacha, las manos permanecen alejadas para luego juntarse al final del movimiento de golpeo, con el fin de permitir que el impulso que tiene la cabeza del hacha haga el trabajo. En cualquiera de estas acciones las manos están juntas, creando un punto sobre el que pivota el extremo pesado del implemento que se utiliza.

De modo diferente a los palos de golf, bates de béisbol y hachas, que son más pesados en el extremo opuesto desde el que se sostienen, una buena espada larga (katana) o la espada de iaido tiende a ser más pesada hacia la empuñadura, con un punto de equilibrio cercano al que la utiliza, aproximadamente a dos tercios de su longitud total desde la punta. En vez de golpear, nuestro objetivo para llegar a cortar con una espada larga es llegar con la parte media y ancha de la espada (koshi) y seguir con un movimiento de corte. La presión descendente a lo largo del filo y el movimiento horizontal de la hoja son importantes. La separación de las manos en la empuñadura permite mantener el control de la hoja de modo que su ángulo permanezca consistente a lo largo del corte mientras la fuerza corporal se transmite hacia todo el filo de la hoja. La coordinación de estos dos componentes hace que sea posible un corte eficiente.

El efecto de palanca no es la razón fundamental para la separación que mantienen las manos. Muchas espadas que se ofrecen a la venta actualmente tienen empuñaduras que exceden las 10 pulgadas [más de $25 \mathrm{~cm}$.] de largo, hasta tanto como 14 pulgadas [aprox. 35,5 cm.]. Los anunciantes sugieren que la empuñadura larga ayuda a aumentar el efecto de palanca, y por tanto la fuerza del corte. Por razones que son muy técnicas, un corte descendente correcto no puede realizarse con una empuñadura demasiado larga. Si usted está considerando comprar una espada de práctica o una espada cortante (shinken) para la práctica del iaido, busque una empuñadura que tenga aproximadamente 9 pulgadas [aprox. $23 \mathrm{~cm}$.] de largo para la práctica del iaido, a menos que sus manos sean excepcionalmente largas. Si sus manos están juntas incluso cuando trata de tenerlas lo más alejadas posible en la empuñadura, encuentre una espada cuya empuñadura sea justo lo suficientemente larga como para dejar una pulgada de distancia $[2,54 \mathrm{~cm}$.] entre ellas.
Figura 1

Advierta que las manos están alejadas todo lo posible en la empuñadura, de modo que haya espacio entre ellas.

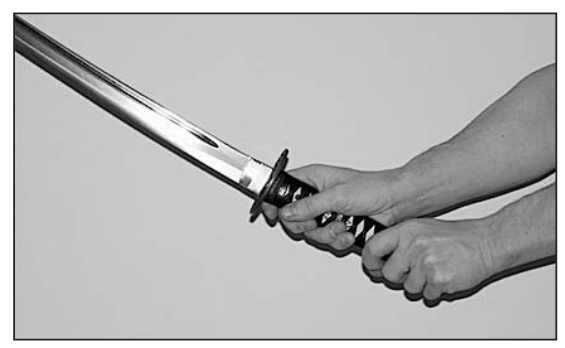

Suino ejecuta el bloqueo inferior en la forma Yaegaki (Las Puertas de las Ocho Veces) del Iaido Eishin-Ryu.

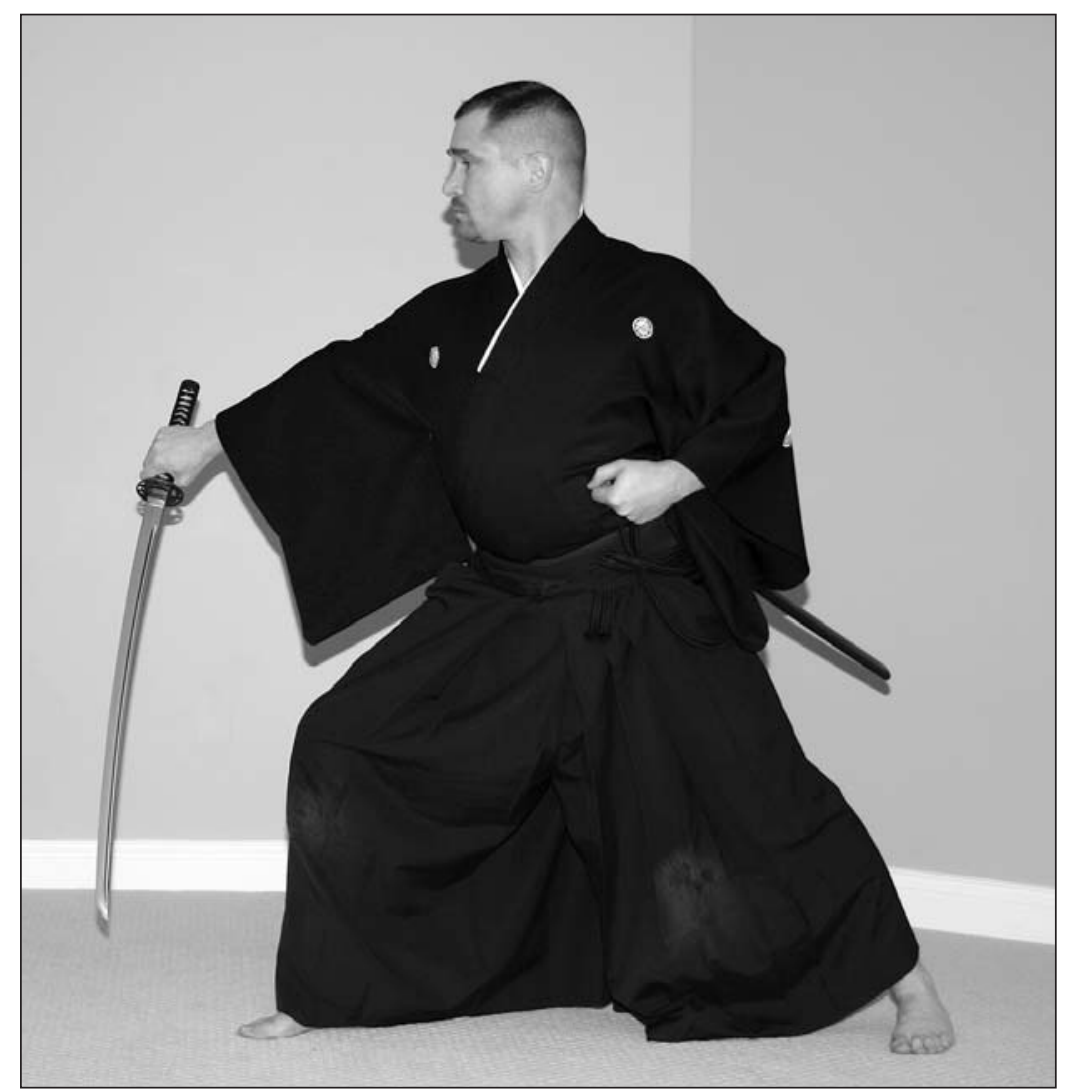




\section{Rotación de las manos}

Durante el primer tercio del corte, las manos rotan ligeramente hacia dentro para llevar las palmas sobre la parte superior de la empuñadura. Este movimiento de las manos hacia adentro continúa, en una extensión más pequeña, a lo largo de los dos tercios finales del corte. Al final del corte se debería tener una sensación de empujar hacia abajo la empuñadura con las manos. Fíjese en la Figura 1 cómo la base de las manos están rotadas o presionando hacia abajo la parte superior de la empuñadura.

Otra forma de pensar sobre la rotación de las manos es considerar dicha rotación como un sub-producto del movimiento de los codos. Mientras la espada se levanta sobre la cabeza, los codos se flexionan y abren hacia los lados. Este movimiento empuja la base de la mano lejos de la empuñadura. Cuando comienza el corte, los codos se extienden se modo que la base de la mano vuelven a empujar sobre la parte superior de la empuñadura.

Muchos estudiantes de iaido exageran la rotación. Una de las expresiones típicas utilizadas por los profesores de iaido es que las manos deberían rotar como si se "retorciese una toalla para sacarle el agua". Los estudiantes que escuchan esta analogía realizan frecuentemente una rotación excesiva, rotando sus muñecas hacia dentro cuando tienen la espada sobre la cabeza, y empleando demasiada rotación durante el corte. Este movimiento excesivo impide que los estudiantes tengan las sensaciones del filo y el control de la hoja.

En vez de esto, los dedos deben moverse muy poco en relación a la empuñadura durante el corte. La mayoría del movimiento que se logra mediante la "rotación" de las muñecas debe suceder en la base de las manos, las cuales, como se ha señalado, están alejadas de la empuñadura cuando la espada está sobre la cabeza, y vuelven a la empuñadura durante el primer tercio del corte. Resulta crucial enfatizar, sin embargo, que las palmas deberían finalizar virtualmente en el borde superior de la empuñadura. Muchos instructores de iaido pueden determinar la habilidad de un estudiante observando simplemente su te-no-uchi. Un estudiante pobremente entrenado sostendrá la espada con las palmas en los lados o en la parte inferior de la empuñadura.

\section{Posición de las muñecas}

Las muñecas deben estar giradas hacia arriba en sentido de los dedos pulgares. Para comprender mejor esta posición, sostenga su brazo derecho en línea recta con su mano extendida hacia delante como si fuese a estrechar la mano a alguien. Sus dedos deben apuntar rectos hacia delante con su pulgar hacia arriba y el meñique hacia abajo, con sus muñecas en una posición neutral. Sin cambiar la posición del

Figura 2

Manos levantadas y retrasadas.

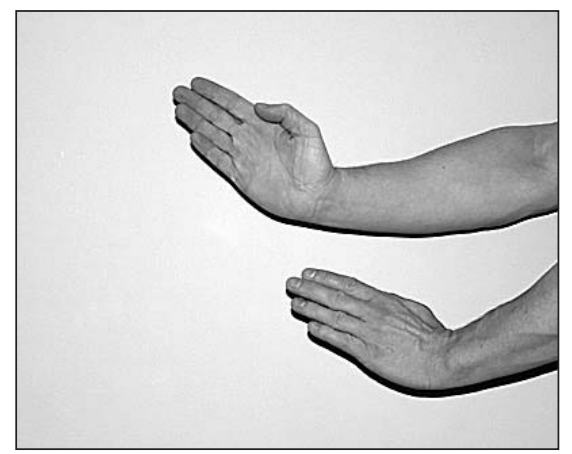

\section{Figura 3}

Posición de las muñecas para el corte descendente.

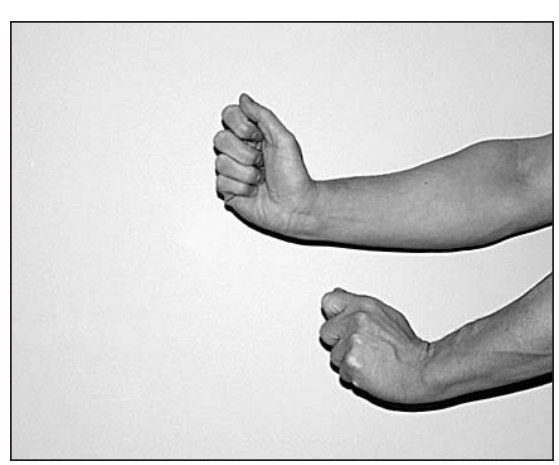




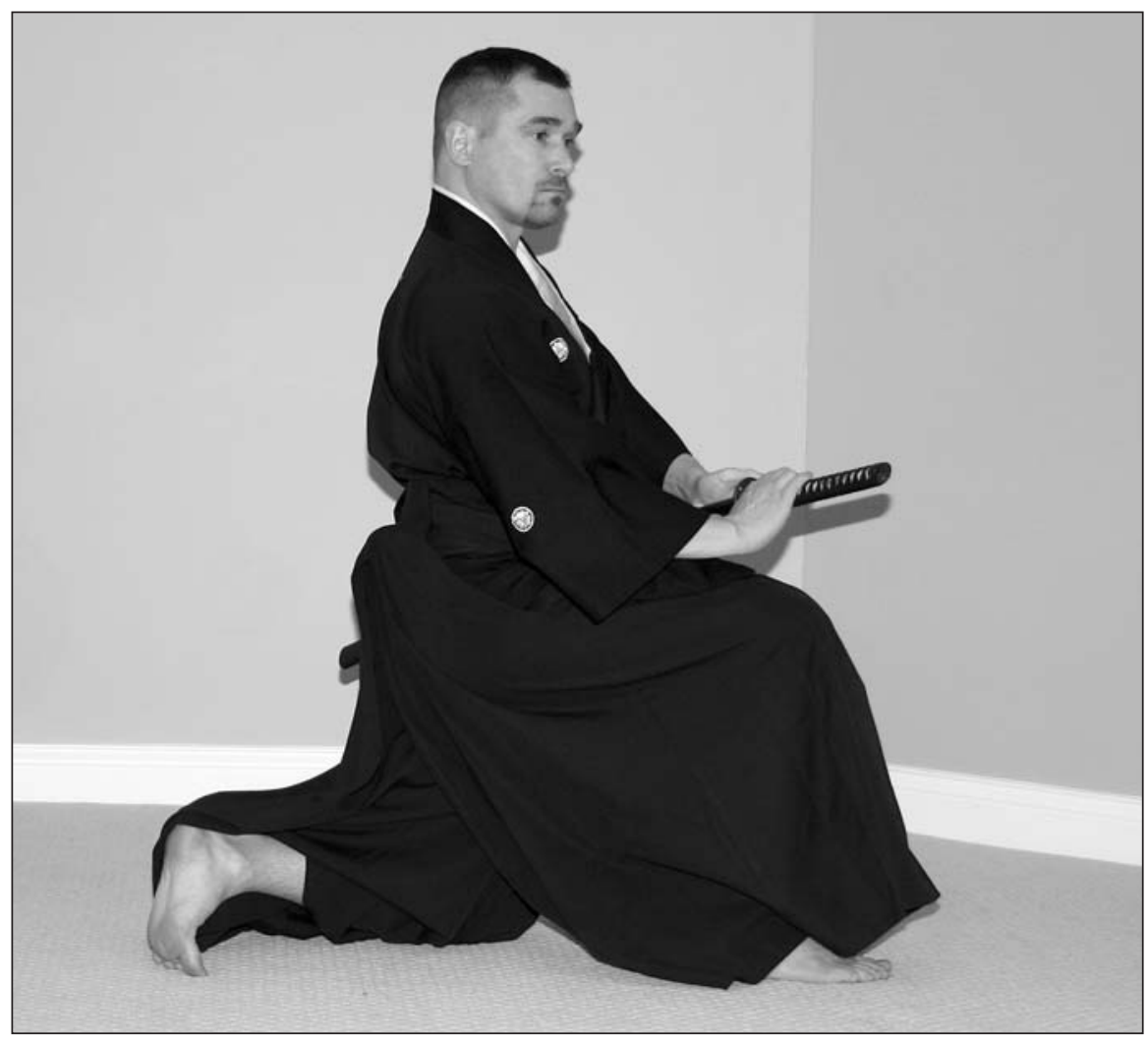

antebrazo, apunte con sus dedos hacia arriba. Se encontrará con que probablemente su constitución anatómica provocará que su muñeca llegue hasta un ángulo de aproximadamente 45 grados.

Deje suelto el puño. Ahora lleve la muñeca hacia atrás, trayendo los nudillos. Esta es la posición aproximada de las muñecas desde el final del primer tercio del corte descendente hasta el final del corte.

El propósito de esta posición de muñecas es doble: primero, junto con la posición extendida de las manos (que se explica más adelante), las muñecas dobladas ayudan a maximizar la cantidad de contacto entre las manos y la empuñadura, añadiendo control sobre la hoja por esta razón. Segundo, la posición ayuda a asegurarse que la parte central más baja de la mano presione sobre la parte superior de la empuñadura. Esto elimina cualquier flexión que pueda estar causada por un puño suelto, haciendo que la hoja sea efectivamente una extensión de los brazos.

\section{La posición de la empuñadura en las manos}

Al final del corte descendente, la parte superior de la empuñadura debe dibujar una línea en las palmas de las manos que se extiende desde la base de la mano, a lo largo del centro de la palma, hasta la base del dedo índice /el extremo distal del primer metacarpo.

Esto proporciona la máxima superficie de agarre posible y proporciona también un fuerte apoyo anatómico para el movimiento de corte. Para lograr esta posición, el estudiante de iaido debe emplear la posición extendida de las manos en la empuñadura.

\section{La posición extendida de las manos}

El agarre en la empuñadura no puede ser un agarre cuadrado tal como el que uno emplearía para agarrar una pesa o un remo. En vez de esto, tal y como se explicó anteriormente, las muñecas deben estar flexionadas hacia los pulgares. Esto permi-
La mano derecha sujeta la empuñadura de la espada desde el lateral para comenzar la extracción inicial de la espada. Esto asegura que la mano esté en la posición correcta para dirigir la fuerza a través de la hoja.

\section{Figura 4}

Línea de contacto de la parte superior de la empuñadura en la palma de la mano.

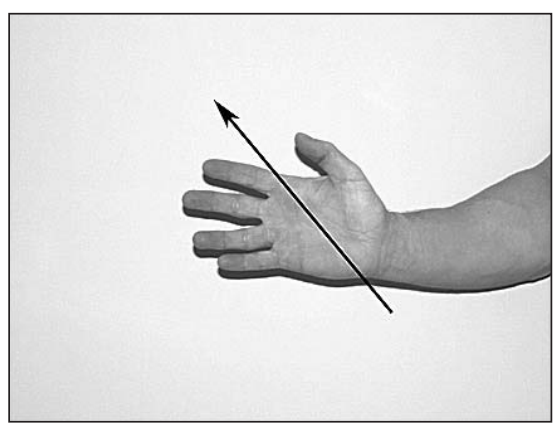

\section{Figura 5}

Muestra un agarre "cuadrado" (incorrecto).

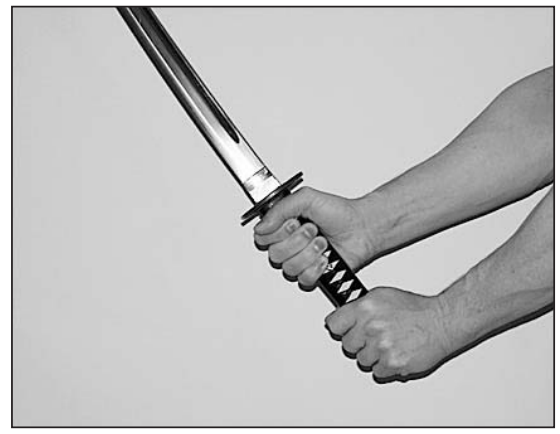

Figura 6

Muestra un agarre extendido (correcto).

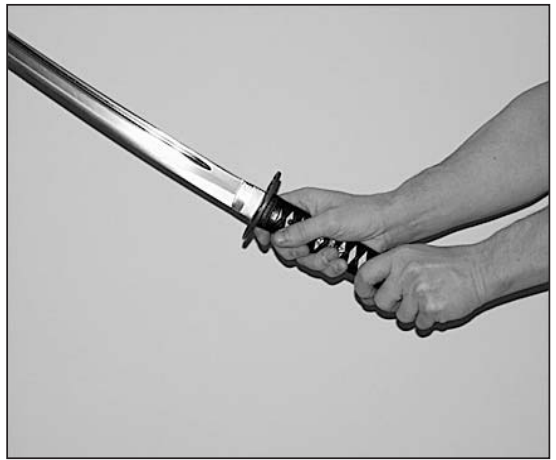


te que los dos dedos largos de cada mano se abran y extiendan ligeramente, sumándose al contacto total entre las manos y la empuñadura, añadiendo así sensación de filo y control de la hoja. Más aún, las muñecas deben estar también flexionadas hacia atrás ligeramente, permitiendo que las bases de las manos se extiendan hacia delante. Tal como se señaló, esto ayuda a dirigir el centro de la parte baja de la palma de la mano hacia la parte superior de la empuñadura, mejorando la transmisión de la energía hacia la hoja.

\section{La sensación en los dedos durante el corte}

La discusión hasta aquí se ha centrado en las manos sobre la parte superior de la empuñadura y en su papel dirigiendo la fuerza a través de la hoja. Sin embargo, la parte inferior de la empuñadura es también importante ya que proporciona retroinformaciones sobre la localización y ángulo del filo de la hoja. La espiga es la extensión de la hoja dentro de la empuñadura. Agarrando la empuñadura, la parte interior de los dedos del practicante le dan la retroinformación que necesita para sentir el ángulo del filo y guiarlo exactamente en su trayectoria de corte. Para muchos de nosotros, la parte interior del segundo nudillo de cada dedo se alineará con la parte inferior de la empuñadura.

Es importante para el estudiante cultivar la sensibilidad de la posición del

El corte de apertura de la forma Tozume (Bloqueado en la Puerta) del conjunto Okuiai (formas secretas).

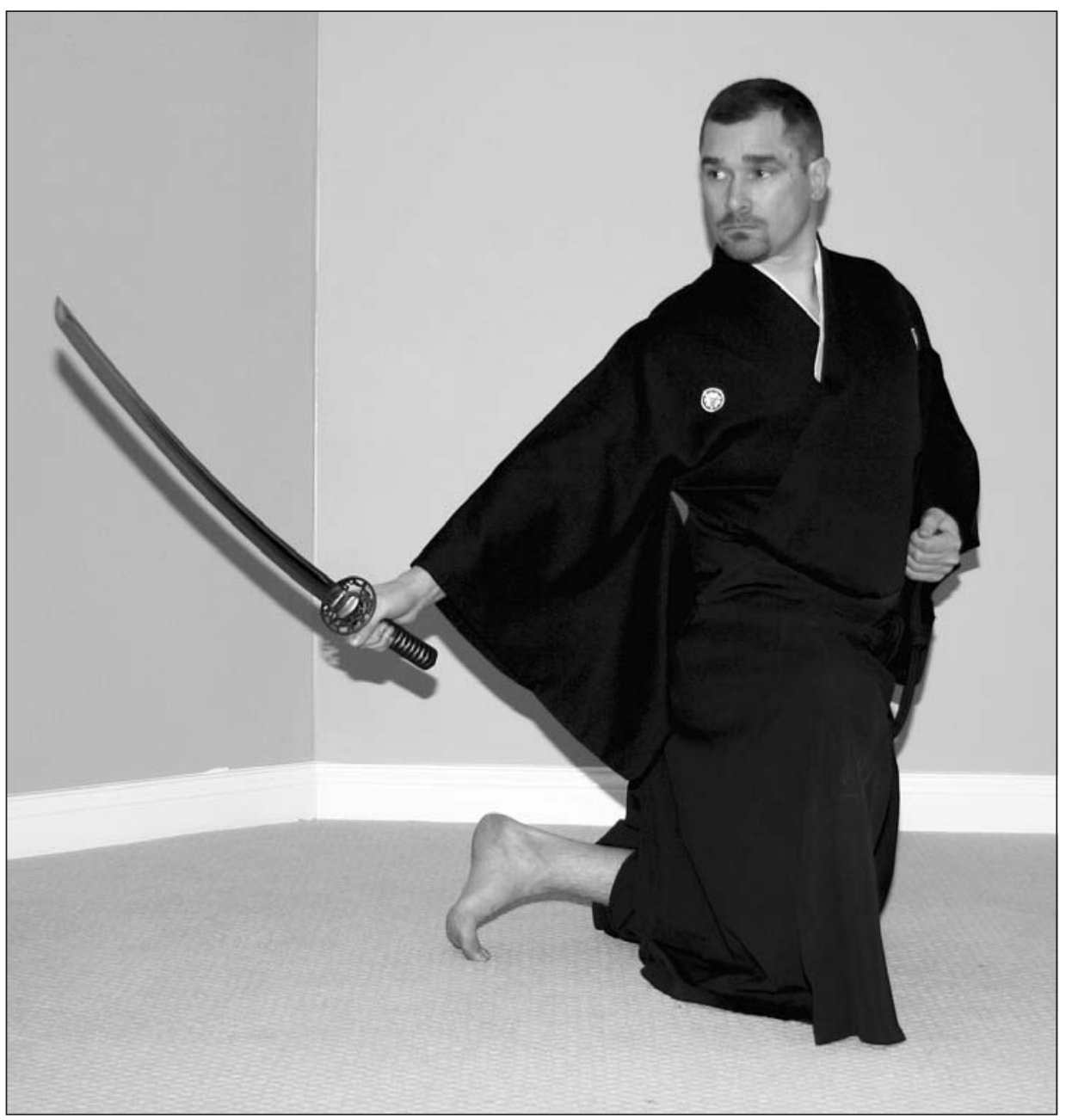
filo. Una buena forma de hacerlo es practicar cortes descendentes mientras se piensa en empujar la hoja hacia abajo con la parte interior de los dedos. Este ejercicio debe repetirse muchas veces. A menudo yo hago esto durante la práctica suburito (cortes repetidos con una espada pesada de Madera o bokken), pero se requiere un bokken que dé muchas sensaciones de hoja, más que uno que sea simplemente largo y pesado. Una vez que se consigue la sensación de guiar el filo con la parte interior de los dedos, las formas completas (waza) deben practicarse tratando de tener en mente estas mismas sensaciones.

Usted también puede usar las pruebas de corte para profundizar en su conocimiento de este aspecto del te-no-uchi. Una vez que comprenda las sensaciones de empujar con los dedos sólo necesitará refrescarlas ocasionalmente, concentrándose en el empuje con los dedos durante el corte. Si usted se concentra en dominar este aspecto sutil del agarre, sus cortes serán más fuertes y mucho más exactos para todo el resto de su trayectoria en el iaido. Eventualmente, cuando guíe el filo correctamente con la parte interior de sus dedos, será posible que usted pueda cortar incluso gruesas esteras prácticamente sin esfuerzo. 


\section{La fuerza del agarre}

La empuñadura debe ser cogida con un agarre firme pero suave. Puede imaginar que es el cuello de un ganso que no quiere dejar escapar, pero que tampoco quiere estrangular. Los dos dedos más pequeños de cada mano deben ser los que agarren con mayor firmeza, mientras que los dos dedos más largos deben agarrar suavemente para permitir que la espada "respire". Aunque los dedos más largos agarran con ligereza, sin embargo, no abra los dedos o permita que se extiendan más allá del canto de la guarda. Cualquier cosa más allá del límite de la guarda es un riesgo de poder ser cortado durante la esgrima.

Un agarre apropiado guía la espada con exactitud y transmite la fuerza del cuerpo a través del filo durante toda la parte efectiva del corte. Al mismo tiempo, un agarre muy apretado evita que la espada se mueva completamente en su arco natural, haciendo que los cortes sean débiles. Cuando los estudiantes "musculares" del iaido realizan las pruebas de corte mientras aprietan la espada demasiado fuerte, sus cortes fallan a menudo. Sólo cuando se relajan y aprenden a guiar la espada suavemente a través de las esteras de paja (tatami), comienzan a cortar limpia y consistentemente. Usted podrá ver que los practicantes habilidosos mueven sus espadas en una trayectoria limpia y directa, sin tensión, y que los movimientos finalizan rápidamente sin muchas vibraciones.

\section{Resumen}

Durante el corte descendente las manos deben colocarse tan lejos una de otra como se pueda, mientras se mantienen los dedos en la empuñadura. Las bases de las manos deben rotar sobre la parte superior de la empuñadura durante el corte, y las muñecas deben estar dobladas hacia arriba en sentido de los pulgares y hacia atrás. La parte superior de la empuñadura debe describir una línea desde la base de la mano, a lo largo de la palma, hasta la base del dedo índice. Las manos deben adoptar una posición extendida en la empuñadura. Los estudiantes de iaido deben cultivar un sentimiento de empuje del filo de la hoja con los dedos a lo largo de los cortes. Los dos dedos pequeños de cada mano han de agarrar firmemente y los dos dedos más largos deben agarrar con suavidad.

En combinación con otras habilidades de movimiento requeridas para dominar el iaido, estos puntos de referencia ayudan a transmitir la energía desde el cuerpo al filo. Practicando cada uno de estos puntos con la ayuda de un buen profesor, los estudiantes mejorarán gradualmente la fuerza y precisión de sus cortes. Más aún, la introspección que se requiere para analizar y aplicar estos puntos de control ayuda a mejorar la concentración y a profundizar en la comprensión del arte. No hay nada que sustituya la práctica concentrada a lo largo del tiempo, y esperamos que estas directrices le ayuden a avanzar correcta y rápidamente hacia el dominio del bello y sutil arte japonés de la esgrima.

\section{Referencia}

Suino, N. (1994). How to watch iaido. Journal of Asian Martial Arts, 3(3): 84-91. 